\title{
Técnica de zona neutra en la rehabilitación de pacientes con el reborde alveolar severamente reabsorbido
}

\section{Neutral zone technique in the rehabilitation of patients with the severely resorbed alveolar ridge}

Melania Ruth Rosado Ventura ${ }^{\text {la }}$

ID https://orcid.org/0000-0002-5143-1391

${ }^{1}$ Investigador independiente.

${ }^{a}$ Cirujano Dentista.

\begin{abstract}
Resumen
El objetivo del presente reporte de caso clínico fue mejorar la retención y estabilidad de la prótesis total. Se presentó el siguiente reporte de caso clínico: Paciente de sexo masculino de 56 años de edad con el reborde alveolar inferior severamente reabsorbido, que acude a la consulta de la Clínica Odontológica de la Universidad Nacional Jorge Basadre Grohmann. Se realizó la confección de la prótesis total inferior con técnica de zona neutra; utilizando compuesto de modelar de baja fusión, moldeándolo en forma de herradura y colocándolo sobre las retenciones de la placa base inferior. Se plastificó con el fuego del mechero; colocando vaselina en la superficie del rodete inferior, como también en los labios y comisura del paciente. Se procedió a colocarlo en la cavidad bucal del paciente indicándole que realice movimientos de succión, contracción de los labios, movimientos de la lengua hacia la derecha e izquierda, y deglución, con la finalidad de registrar en el rodete la acción muscular para determinar la localización exacta de la zona neutra hasta que la godiva endurezca. La placa base de rodete de modelina fue retirada de la cavidad bucal para examinarla minuciosamente. Se repitió el mismo procedimiento tres veces hasta obtener un registro correcto; teniendo mejores resultados de lo que brindan las prótesis convencionales. En conclusión, la técnica de zona neutra es útil en pacientes con rebordes alveolares severamente reabsorbidos; demostrando ser eficiente, mejorando la retención y estabilidad de la prótesis total.
\end{abstract}

Palabras clave: Zona neutra, reborde alveolar, prótesis dental total.

\begin{abstract}
The aim of this clinical case report was to improve the retention and stability of the total prosthesis. The following clinical case report was presented: 56-year-old male patient, with the severely resorbed lower alveolar ridge, who visits the Jorge Basadre Grohmann National University Dental Clinic. The lower total prosthesis with neutral zone technique was made, using a low-melt modeling compound, molding it into a horseshoe shape and placing it on the retentions of the lower base plate, the prosthesis was plasticized with the lighter fire, placing petroleum jelly on the surface of the lower impeller as well as on the lips and commissure of the patient. It was placed in the patient's oral cavity and instructed to perform suction movements, lip contraction, tongue movements to the right and left, and swallowing, in order to register the muscle action on the impeller to determine the exact location of the neutral zone until the godiva hardens. The modeline impeller base plate was removed from the oral cavity to examine it thoroughly. The same procedure was repeated three times until a correct registration was obtained. Resulting in better than conventional prosthetics. In conclusion, this neutral zone technique is useful in patients with severely resorbed alveolar ridge, proving to be efficient, improving the retention and stability of the total prosthesis.
\end{abstract}

Keywords: Neutral zone, alveolar ridge, total dental prosthesis. 


\section{Introducción}

Se le llama zona neutra "Al espacio potencial entre los labios y mejillas por un lado y la lengua en el otro; esa área o posición es donde las fuerzas entre la lengua, mejillas y labios son iguales" (1).

Roberts lo llamó el "espacio potencial", mientras que otros lo llamaron "espacio muerto", "zona de conflicto mínimo", "espacio de prótesis", "zona recíproca" y "zona de función muscular neutra" (2-4).

El tratamiento habitual a la condición de desdentado total es el uso de la prótesis completa removible, que, en general, responde satisfactoriamente a los requerimientos funcionales en el maxilar superior; en cambio en la mandíbula, por razones anatómicas y fisiológicas, condicionan un dispositivo con insuficientes propiedades de retención, soporte y estabilidad; lo que da origen a dificultades severas en la masticación, y una permanente sensación de inseguridad para mantener la adaptación de la prótesis a los tejidos orales (5-7). Como resultado se afecta la autoestima, se limita la interacción social, compromete la nutrición; lo que se traduce en detrimento de la calidad de vida (8).

La rehabilitación oral de un paciente edéntulo total se basa en parámetros anatómicos conservados; buscando siempre la armonía estética y funcional de la dentadura con los tejidos orales y periorales (9).

La zona neutra es, además, un área dinámica, modificada en el tiempo; especialmente por la continua reabsorción del hueso del reborde residual, y por cambios en las fuerzas de la musculatura paraprotética (10). Este espacio no necesariamente se ubica sobre la cresta del reborde hacia lingual o bucal de él. Su ubicación dependerá de la musculatura protésica, la cual varía entre los pacientes, y no necesariamente corresponde con la posición mecánica de los dientes protésicos, debido principalmente a los diversos patrones de reabsorción del reborde alveolar en los diferentes segmentos maxilares. También, se debe destacar que existen diferencias individuales que comienzan a establecerse desde la erupción de los dientes naturales, los cuales están expuestos a influencias ambientales conocidas $(11,12)$.

La estabilidad de las prótesis totales está influenciada por el sistema neuromuscular circundante en la cavidad oral; la retención y la estabilidad se vuelven desfavorables cuando la reabsorción de los rebordes alveolares se torna más severa, especialmente en la mandíbula.
Este informe detalla el caso de un paciente de sexo masculino, de 56 años de edad, con reborde alveolar inferior severamente reabsorbido; que acude a la clínica Odontológica de la Universidad Nacional Jorge Basadre Grohmann, donde se realizó la confección de su prótesis total inferior con técnica de zona neutra; en equilibrio muscular, a través de contornos óptimos de la prótesis y fisiológicamente apropiados.

El objetivo de este reporte de caso fue mejorar la retención y estabilidad, mediante la confección de la prótesis total inferior con técnica de zona neutra, del reborde alveolar inferior severamente reabsorbido.

\section{Caso clínico}

Paciente de 56 años de edad, de sexo masculino requiere tratamiento de prótesis total. Se realizó la historia clínica; durante la anamnesis, el paciente refiere haber padecido de fiebre reumática. En el examen clínico observamos a un paciente de tez trigueña con simetría facial, sin lesiones ni adenopatías aparentes. Al examen clínico intraoral presenta reborde alveolar inferior severamente reabsorbido (clase $\mathrm{V}$ de Atwood); forma triangular; reborde alveolar superior reabsorbido (clase $\mathrm{V}$ de Atwood); forma triangular; carrillos de coloración negruzca bilateral (Fig. 1: a, b, cyd).

Al examen radiográfico se observa rebordes alveolares severamente reabsorbidos; conducto dentario inferior cercano al reborde alveolar (Fig. 2).

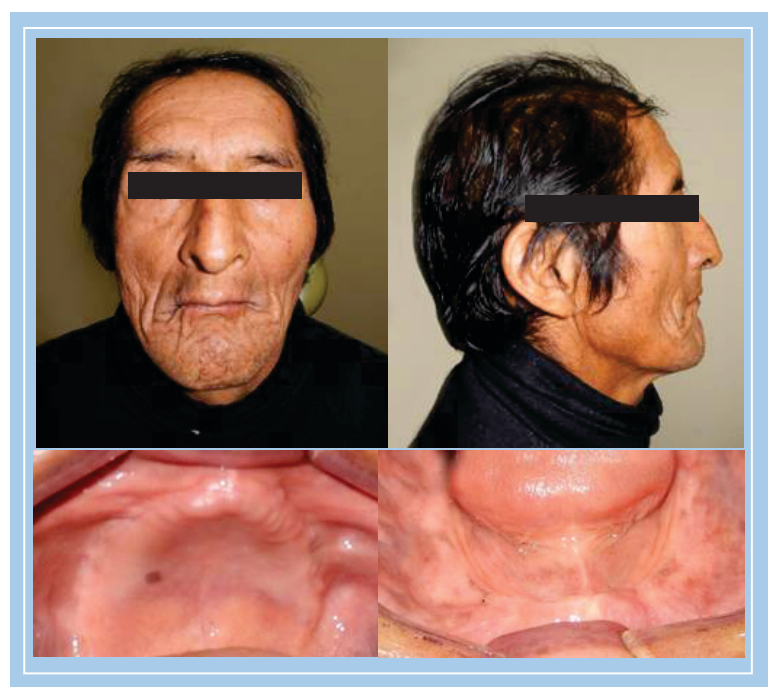

Figura 1. (a) Vista extraoral frontal, (b) Perfil anteroposterior, (c) Maxilar superior, (d) Maxilar inferior.

Fuente: E.P. 


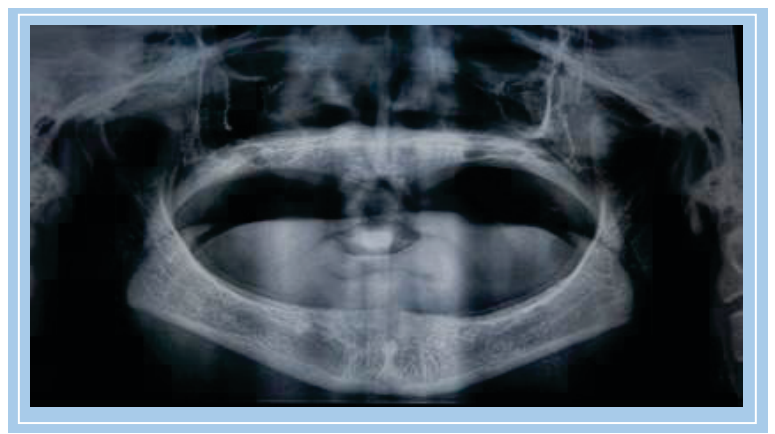

Figura 2. Radiografía panorámica.

Fuente: E.P.El diagnóstico fue: edéntulo total superior (clase V Atwood) y edéntulo total inferior (clase V Atwood).

El tratamiento realizado fue dos prótesis totales; la superior con técnica convencional y la inferior con técnica de zona neutra. Se tomaron impresiones primarias con alginato para la confección de cubetas individuales, luego se continuó con el sellado periférico, y la toma de impresiones secundarias con pasta zinquenólica con el paciente sentado a $90^{\circ}$ (figura 3: a, b, c, d, e y f). Se realizó el ribeteado y encajonado para obtener los modelos de trabajo; en ellas se confeccionó las placas base; en el caso de la arcada inferior se usó modelina tipo I marca Perfectín. Se confeccionó una tira de compuesto de modelar de baja fusión moldeándolo en forma de arcada; la cual se colocó sobre las retenciones de la placa base inferior para poder fijar los bordes terminales de este rodete a base de godiva, luego se procedió a plastificarlos con el fuego del mechero, colocando vaselina en la superficie del rodete inferior, como también en los labios y comisura del paciente. Se procedió a colocar la placa base con rodete de godiva en la cavidad bucal del paciente indicándole que realice movimientos de succión; contracción de los labios; movimientos de la lengua hacia la derecha e izquierda, y deglución, con la finalidad de registrar en el rodete la acción muscular para determinar la localización exacta de la zona neutra hasta que la godiva endurezca (Fig. 4). La placa base de rodete de modelina fue retirada de la cavidad bucal para examinarla minuciosamente, descartando desgarros, o fractura del material, que pudieran alterar el reporte del registro de la zona neutra. Se repitió el mismo procedimiento 3 veces, hasta obtener un registro correcto, retirando los excesos con bisturí.

Luego se procedió a confeccionar la placa base con rodete de cera para la arcada superior con la técnica convencional (Fig. 4: a, b, c, d, e y f). Se determinó el plano de oclusión, primero en el rodete inferior. Teniendo como referencia el borde del labio y la comisura del labio inferior, se eliminó excesos, y se observó que el plano de oclusión quede a nivel del borde periférico de la lengua en reposo. Se determinó la altura del rodete pidiéndole al paciente que cuente del 1 al 20; que diga palabras como "Misisipi"; que humedezca los labios con la punta de su lengua para comprobar la retención y estabilidad; luego se determinó el plano de oclusión de rodete superior teniendo como referencia que el plano oclusal anterior del rodete superior quede 2 $\mathrm{mm}$ por debajo del borde inferior del labio superior; colocando el plano de Fox paralelo al plano de Frankfort, y el plano oclusal paralelo al plano de Camper; adaptándose al espacio que deja el rodete inferior. Se determinó la dimensión vertical utilizando la técnica de fonación y de deglución. Se le pidió al paciente que pronuncie palabras como "Misisipi" o "foco", y que degluta, observando $1 \mathrm{~mm}$ de espacio entre ambos rodetes. Se determinó el relleno facial en base al rodete inferior. Se tomó el registro con el arco facial con el paciente sentado en $90^{\circ}$, y se hizo el montaje del modelo superior en ASA, luego se procedió a tomar el registro en relación céntrica, colocándole dos rollos de algodón a nivel de los premolares en los rodetes por 7 minutos. Se observó que la mandíbula no ofrecía resistencia al guiarla. Se colocó una fina capa de pasta zinquenólica en el rodete superior y se tomó el registro en relación céntrica. Se hizo el montaje del modelo inferior. Se elaboraron llaves de silicona de masa pesada sobre el rodete inferior colocado en el modelo de trabajo (Fig. 5: a, b, c y d). Se seleccionó el color y tamaño de dientes. Se efectuó el enfilado dentario con dientes monoplanos guiado por la localización de la zona neutra. Se hizo la prueba de enfilado en boca, verificando la dimensión vertical y la estabilidad y retención. Se observó una correcta intercuspidación, garantizando el éxito del tratamiento dirigido a mejorar la estabilidad y la retención (Fig. 6). Se hizo el enmuflado, acrilizado y el remontaje para el ajuste oclusal de los dientes (Fig. 7); luego se instalaron las prótesis totales en boca del paciente, comprobando la retención y estabilidad; pidiéndole al paciente que realice movimientos funcionales y de fonación. Se hizo el ajuste oclusal con papel articular (Fig. 8), luego se programó el primer control a las 24 horas, después a los 3 días, a la semana y al mes. Se informó al paciente de la importancia de la higiene, cuidados y mantenimiento de sus prótesis (Fig. 9).

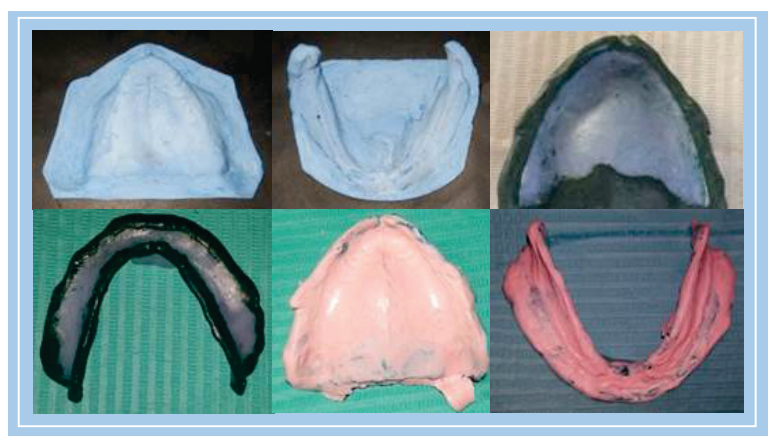

Figura 3. (a) Modelo de estudio superior, (b) Modelo de estudio inferior, (c) Sellado periférico superior, (d) Sellado periférico inferior, (e) Impresión secundaria superior, (f) Impresión secundaria inferior.

Fuente: E.P 


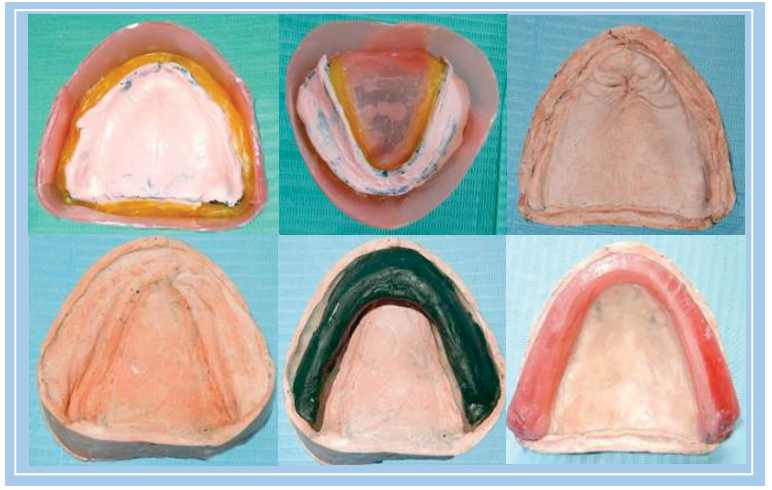

Figura 4. (a) Ribeteado y encajonado superior, (b) Ribeteado y encajonado inferior, (c) Modelo de trabajo superior, (d) Modelo de trabajo inferior, (e) Placa base con rodete de modelina inferior técnica Zona Neutra, (f) Placa base con rodete de cera superior.

\section{Fuente: E.P.}

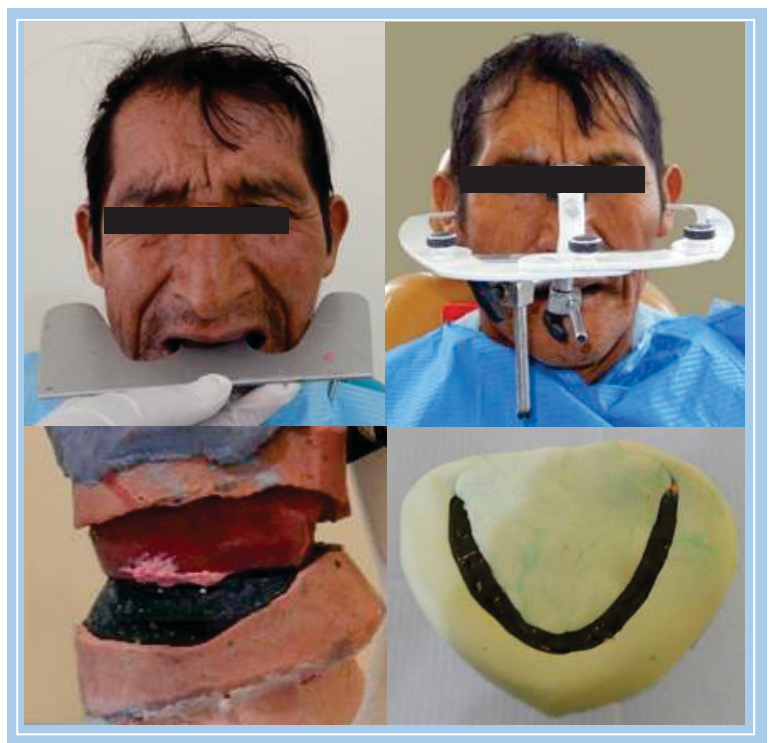

Figura 5. (a) Determinación del plano de oclusión, relleno facial y dimensión vertical, (b) Registro con el arco facial, (c) Montaje de modelos en ASA en relación céntrica, (d) Llaves de silicona. Fuente: E.P.

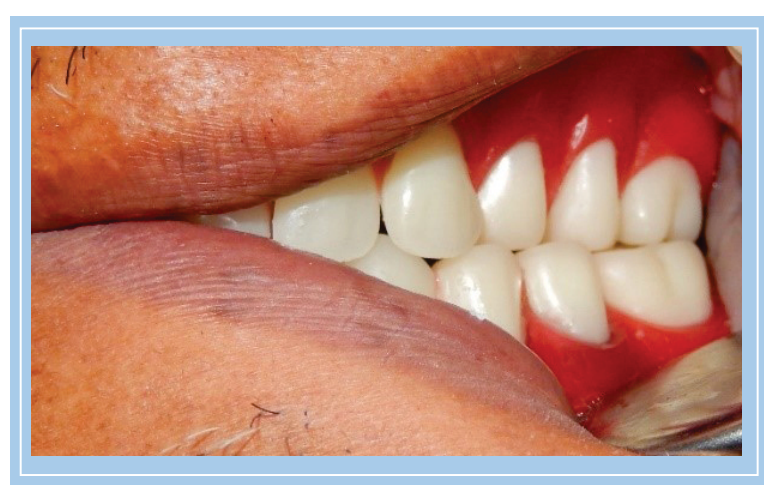

Figura 6. Prueba de enfilado.

Fuente: E.P.

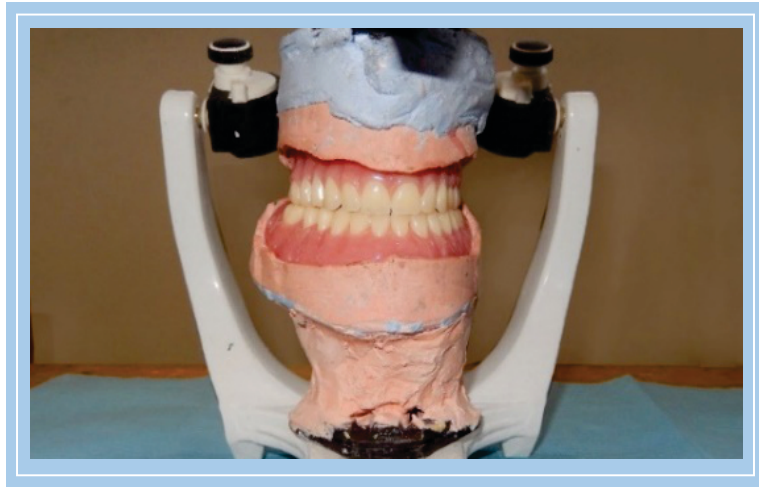

Figura 7. Remontaje y ajuste oclusal.

Fuente: E.P.

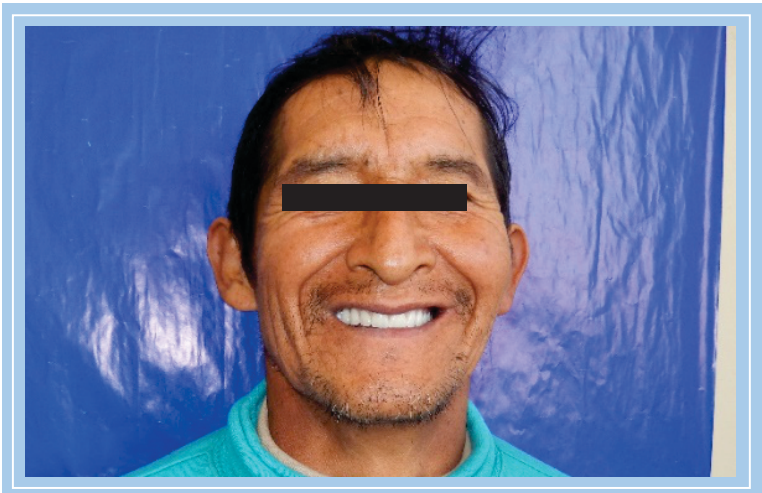

Figura 8. Instalación de prótesis.

Fuente: E.P.

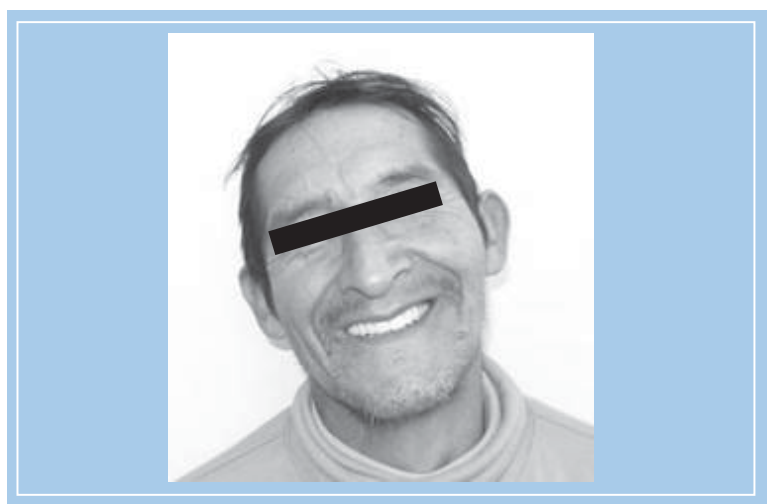

Figura 9. Control después de 24 horas.

Fuente: E.P.

\section{Discusión}

Este artículo busca poder enriquecer y ampliar nuestros conocimientos sobre esta técnica, asimismo, contribuir en las líneas de la investigación. La confección de la prótesis total inferior del reborde alveolar severamente reabsorbido con técnica de zona neutra permite resultados beneficiosos para el paciente; mejorando la retención y estabilidad, elaborando una prótesis total en 
equilibrio muscular. La técnica de zona neutra utilizada en el presente estudio difiere de la técnica convencional, debido a que registra la dinámica fisiológica de la función muscular, oral y perioral de manera simplificada, mejorando así la retención y estabilidad de la prótesis total en pacientes con rebordes alveolares severamente reabsorbidos.

Saravanakumar et al. (13) describen, en su estudio, la confección de prótesis totales con técnica de zona neutra a un paciente con mala coordinación muscular y con la cresta mandibular atrófica; mejorando la retención y estabilidad de las prótesis; logrando el éxito del tratamiento con la eficiencia masticatoria, comodidad y la estética del paciente, concordando con los resultados de este estudio de reporte de caso clínico.

Astorga et al. (14), en su estudio de experimentación clínica con un paciente que presentaba un reborde óseo reducido mandibular, y que padecía de espasmos musculares, optaron por el uso de la técnica de zona neutra mediante un registro piezográfico de la misma; con lo cual se obtenía una zona en la que las fuerzas musculares se contrarrestaban, y de esta manera se conseguía una mejor relación entre la prótesis y los tejidos que la rodean; con lo que el tratamiento brindaba un mayor confort para el paciente, siendo mucho más estable y retentivo, además de que presentó excelente resultado en la fonética del paciente y la comodidad con su prótesis. Los resultados de estudio coinciden con el reporte de caso clínico de Saravanakumar.

Bustillos et al. (15), en su estudio, realizaron un registro piezográfico de la zona neutra, donde se obtuvo una reproducción apropiada de la relación entre superficie protésica externa y los tejidos que se encuentran en la periferia, aumentando la retención y la estabilidad del aparato protésico insertado en la cavidad bucal. La dentadura construida bajo esta técnica demostró mayor grado de comodidad atribuida a que no interfiere con las funciones de los labios, mejillas y lengua. Sí existe coincidencia con nuestro estudio.

Anampa M. (9), en su informe de caso clínico, describe la técnica de zona neutra modificada para la rehabilitación oral de un paciente edéntulo total inferior cuyo reborde presentó un severo grado de reabsorción. Consiguiendo devolver la armonía entre la prótesis y la musculatura peribucal, lográndose la retención y estabilidad de la misma.

\section{Conclusión}

En conclusión, la técnica de zona neutra es útil en pacientes con rebordes alveolares severamente reabsorbidos; demostrando ser eficiente, mejorando la retención y estabilidad de la prótesis total.

\section{Referencias bibliográficas}

1. Keith J., Morgano S. et al., El Glosario de Terminos de Prostodoncia. Glossary of Prosthodontic Terms Committee of the Academy of Prosthodontics, J Prosthet Dent ChicagoUSA, 2017.

2. Roberts AL. The effects of outline and form upon denture stability and retention. Dent Clin Am. 1960;4:293-303.

3. Makzoumé JE. Morphologic comparison of two neutral zone impression techniques: A pilot study. J Prosthet Dent. Beirut, 2004;92:563-8.

4. Fenn HR, Liddelow KP, Gimson AP, MacGregor AR. 3rd ed. London: Wright; 1989.

5. Thalji G, McGraw K, Cooper LF. Maxillary Complete Denture Outcomes: A Systematic Review of Patient-Based Outcomes. Int J Oral Maxillofac Implants. Chicago- USA, 2016;31:169-81.

6. Komagamine Y, Kanazawa M, Kaiba Y, Sato Y, Minakuchi S, Sasaki Y. Association between self- assessment of complete dentures and oral healthrelated quality of life. J Oral Rehabil. Tokyo, 2012; Nov: 39(11):847-57.

7. Prithviraj DR, Madan V, Harshamayi P, et al. A comparison of masticatory efficiency inconventional dentures, implant retained or supported overdentures and implant supported fixed prostheses: a literature review. J Dent Implant. India, 2014;4:153-7.

8. López CS, Saka CH, Rada G, et al. Impact of fixed implant supported prostheses in edentulous patients: protocol for a systematic review. BMJ Open. Chile, 2016; 6: e009288.

10. Ikebe K, Okuno I, Nokubi T. Effect of adding impression material to mandibular denture space in piezography. J Oral Rehabil, 2006; 33: 409415.

11. Agarwal S, Gangadhar P.A Simplified approach for recording neutral zone. J Indian Prosthodont Soc, Indian, 2010;10(2):102-104.

12. Hina Z, Raja M, Nasir S. Neutral zone dentures versus convetional dentures diverse edentulous periods. Biomedica, Pakistan, 2009; 25: 136-145. 
13. Saravanakumar P, Thirumalai S, Thangarajan, Mani U, and Kumar V. Improvised Neutral Zone Technique in a Completely Edentulous Patient with an Atrophic Mandibular Ridge and Neuromuscular Incoordination: A Clinical Tip. J Indian Cureus. 2017. 24 de Apr. 9(4).12.

14. Astorga E, Lee X y Vergara C. Reporte clínico: Registro piezográfico de la zona neutra, en un paciente con espasmos musculares involuntarios. Clin. Periodoncia Implantol. Rehabil. Oral. Chile, 2013.6(3); 134-137.

15. Bustillos L, Velazco G, Bustamante M. Clínica Zona Neutra: Descripción de una técnica en la construcción de dentaduras completas. REDOE. Venezuela. 2017. 15 feb. 10:42.
Correspondencia:

melth1@hotmail.com
Fecha de recepción : 22 de febrero de 2020

Fecha de aceptación : 30 de mayo de 2020 